\title{
Journal of Vasceulanr Researdh
}

Abiona, A. 381

Abraham, D. 383

Acheson, A. 373

Ahmad, Z. 375

Ahmed, A. 385

Andrade, S.P. 386

Aplin, J.D. 385

Ashhurst, D.E. 381

Austin, C. 384

Azarbod, P. 374

Bates, D.O. 377

Bawden, L.C. 390

Belo, A.V. 386

Bennett, A.R. 378

Beraldo, W.T. 386

Bicknell, R. 374

Black, C. 383

Boot-Handford, R.P. 391

Bouchier-Hayes, D. 376

Boulton, M. 385, 386

Boulton, M.E. 390

Brenchley, P.E.C. 387

Brindle, N.P.J. 374

Brookes, Z.L.S. 378

Brown, M.D. 380, 388

Brown, N.J. 378

Brunini, T.M.C. 379

Bujny, T. 379

Bundred, N.J. 390

Caddick, S.L. 383

Campbell, F. 376

Canfield, A.E. 373, 389, 390, 391

Carrasco, G. 391

Chamberlain, J. 389

Chen, C.-P. 385

Church, H.J. 385

Church, M.K. 378

Clark, P. 383, 392, 393

Clark, S. 376

Clough, G.F. 378

Coleman, P.J. 381, 388

Coleridge-Smith, P.D. 382

Cooper, D. 381

Crossman, D.C. 389

Cumberland, D.C. 389

Dewhirst, M.W. 373

Dickson, A.J. 390

Doherty, M.-J. 391
Abstracts of the Joint Meeting of the British Microcirculation Society and the Northern Vascular Biology Group
Donovan, D. 376

Dye, J. 393

Dye, J.F. 383, 392

Egginton, S. 386

Ellory, J.C. 379

Estdale, S.E. 381

Faircloth, C. 390

Fajkumar, V. 383

Feldman, M. 377

Finn, A. 388

Firth, J.A. 383, 392, 393

Foreman, D. 385

Forootan, S.S. 383, 384

Francis, S.E. 389

Gamble, J. 375, 390

Gartside, I.B. 375, 390

Gerber, R.T. 378

Glyn, M.C.P. 376

Gonzalez, C. 391

Graham, A.M. 381

Grewal, P.S. 375

Gunn, J. 389

Haddaway, E. 393

Hampson, I. 387

Hampson, L. 387

Hanssen, H. 379

Harmey, J.H. 376

Harris, N.D. 379,383

Harvey, B. 391

Heagerty, A.M. 372

Heerkens, E. 372

Helliwell, T.R. 383, 384

Herrick, A. 376

Holemans, K. 378

Holt, C.M. 389

Homer-Vanniasinkam, S. 381

Hudlicka, O. 380, 388

Ireland, G. 386

Issa, R. 379

Jablenska, R. 392, 393

Jacob, R. 392

James, D.K. 393

Jayson, M.I.V. 376

Jones, A.S. 383, 384
Kajimura, M. 375

Kale, M. 389

Kaluza, J. 379

Kariappa, S. 393

Kay, E. 376

Ke, Y. 383

Khaliq, A. 385

Kirton, C.M. 376

Kishver, Z. 381

Krupinski, J. 379

Kumar, P. 379

Kumar, S. 379, 387

Lammiman, M. 393

Leach, L. 383, 393

Leake, D.S. 382

Levick, J.R. 374, 381, 388, 389

Li, C.G. 387

Lin, C. 373

McCarthy, M.J. 374

Machado, R.D.P. 386

Maciewicz, R. 377

McLeod, D. 385, 386

McNamara, D.A. 376

Macro, J. 387

Maisonpierre, P. 373

Mann, G.E. 379, 382, 391, 392

Mantell, D.J. 390

Marron, M.B. 374

Mason, R.M. 381, 388

Mawer, E.B. 390

Mendes Ribeiro, A.C. 379

Miah, S. 385

Michel, C.C. $375,380,388$

Milkiewicz, M. 388

Miotla, J. 377

Moffitt, H. 380

Monk, P.N. 388

Montecinos, V.P. 392

Moore, J.L. 386

Moore, T. 376

Morrison, L.C. 390

Mortimer, P.S. 374

Murdoch, C. 388

Nash, G.B. 376

Neal, C.R. 388

Nield, H. 391

Nör, J.E. 372

\section{KARGER}

$$
\text { () } 1998 \text { S. Karger AG, Basel }
$$

Fax +41613061234 E-Mail karger@karger.ch www.karger.com

Accessible online at: http://BioMedNet.com/karger 
Odom, N.J. 387

Ohanian, V. 372

Onwu, N. 387

Otter, D.J. 384

Paleolog, E. 377

Parkin, S.M. 381

Pearce, S. 380

Peters, K.G. 373

Polverini, P. 373

Polverini, P.J. 372

Ponting, J. 387

Porter, J. 382

Poston, L. 378

Rajbhandari, S.M. 379

Rao, P.S. 373

Redziejewski, C. 373

Reilly, C.S. 378

Richards, A.J. 385

Richards, J.P. 382

Roberts, I.S. 387
Roberts, T.E. 387

Rooney, P. 387

Rubin, K. 383

Schor, A.M. 373

Scott, D. 381,388

Scurr, J.H. 382

Shan, S. 373

Shaw, L. 372, 384

Sherman, M. 374

Shoab, S.S. 382

Silgram, H. 380

Siow, R.C.M. 382

Smith, J. 387

Sobrevia, L. 391, 392

Stanton, A.W.B. 374

Steinert, J.R. 392

Sundberg, C. 383

Tarso, A.M. 386

Teixeira, A.S. 386

Tesfaye, S. 379
Thompson, W.D. 380

Tuckwell, D. 373

Van Assche, F.A. 378

Walsh, T.N. 376

Wang, J.-H. 376

Ward, B.J. 376

Ward, J.D. 379, 383

Watson, C.J. 387

Weich, H. 385

Wheeler-Jones, C.P.D. 391

Williams, G. 385

Wong, A. 373

Wood, A.C. 391

Wyatt, A. 391

Yancopoulos, G.D. 373

Young, D. 379

Young, S. 377

Zhou, A.-L. 386 